\title{
The consumption of ultra-processed foods by fish-eaters, vegetarians and vegans is associated with the duration and commencing age of diet
}

Abstract

Introduction: In Western countries, the growing availability of animal products alternatives such as plant-based drinks or vegetarian patties for vegetarians and vegans, suggest that there could be an increase of their consumption of ultra-processed foods (UPFs). However, very little is known about the share of UPFs, and inversely unprocessed foods (UnPFs), in their diet. Thus, the aim of this study was to describe the proportion of UPFs and UnPFs, in adults from the NutriNet-Santé cohort, according to three types of vegetarian diets compared to meat-eaters. The relationship between diet characteristics such as duration and commencing age of the diet, and the share of UPFs, and UnPFs, were also studied among individuals following vegetarian diets.

Materials and Methods: The study population $(n=21,212)$ was divided into 4 groups: 19,812 meat eaters, 646 fish-eaters, 500 vegetarians and 254 vegans. Daily food intakes, adjusted for sex, age and total energy intake, were collected using repeated 24-h dietary records. Vegetarian diets were described according to indicators of UPFs and UnPFs, and the Healthy and Unhealthy plant-based Diet Indices (hPDI/uPDI). In a sub-sample without meat eaters $(n=1,400)$, multivariable linear regression models were performed to assess the association between UPF consumptions and diet characteristics.

Results: UPFs accounted for 33.0\%, 32.5\%, 37.0\% and 39.5\% respectively for meat-eaters, fish-eaters, vegetarians and vegans. On the contrary, UnPFs accounted for $29 \%, 29 \%, 27 \%$ and $31 \%$. The means of uPDI and hPDI indices were respectively equal to 54.0, 53.2, 56.9 and 59.9, and 53.5, 60.6, 61.4 and 67.9 for meat-eaters, fish-eaters, vegetarians and vegans. Concerning diet characteristics, a shorter duration of being vegetarian, or a younger age at commencing of the diet was significantly associated with increased consumptions of UPFs (respectively $\mathrm{p}=0.0010$ and $\mathrm{p}<0.0001$ ).

Discussion: We showed that vegetarians can be distinguished according to the share of both UPFs and UnPFs in their diet, but also concerning the diet quality according to hPDI and uPDI. Thus, the share of UPFs in the diet and associated characteristics such duration and commencing age of the diet, should be considered in future studies about the link between vegetarianisms and health.

\section{Conflict of Interest}

There is no conflict of interest. 